\title{
Characterization of MEPCM-Incorporated Paint as Latent Heat Storage System
}

\author{
Terence P. Tumolva and Noel S. Sabarillo
}

\begin{abstract}
This study aimed to determine the effect of the mixing speed and pre-polymer dropping rate during synthesis of microencapsulated phase change materials (MEPCMs), and to assess the performance of MEPCM-incorporated paint as a latent heat storage (LHS) system. $\boldsymbol{N}$-octadecane as phase change material was encapsulated with resorcinol-modified ureamelamine-formaldehyde at two different mixing speeds and four different pre-polymer dropping rates, and Fourier transform infrared (FTIR) spectroscopy was done to confirm success of microencapsulation. Scanning electron microscopy (SEM) revealed that increasing the homogenization speed and decreasing the pre-polymer dropping rate decreases the microcapsule size. Differential scanning calorimetry results showed that latent heat and encapsulation ratio increases with increasing mixing speed and decreasing pre-polymer dropping rate. The synthesized MEPCMs were incorporated into white paint at three different concentrations, and temperature profiling revealed that the paint's temperature buffering capacity generally increases with increasing mixing speed, decreasing pre-polymer dropping rate and increasing MEPCM concentration.
\end{abstract}

Index Terms - Heat storage, MEPCM, phase change material, microencapsulation.

\section{INTRODUCTION}

Thermal energy storage (TES) systems provide possible means for energy conservation by absorbing or releasing excess heat to the surroundings over a desired period of time, depending on the application. One type of TES, the latent heat storage (LHS), stores and releases heat through phase transition- thus, providing higher storage density even with a smaller temperature difference between absorbing and releasing heat steps [1].

Phase change materials (PCMs) are used in LHS systems because they are capable of storing large amounts of heat without significantly changing the control temperature as they undergo phase transition. PCMs that have been studied in previous researches include hydrated salts, fatty acids, and paraffin waxes (such as $n$-octadecane) [2]. In previous studies, it has been observed that microencapsulating PCMs

Manuscript received December 26, 2016; revised March 25, 2017. This work was supported by the Department of Science and Technology (DOST) under the Engineering Research and Development for Technology (ERDT) Scholarship Program.

Terence P. Tumolva is with the Department of Chemical Engineering, University of the Philippines, Diliman, Quezon City 1101 Philippines (e-mail: tptumolva@up.edu.ph).

Noel S. Sabarillo was with the Department of Chemical Engineering, University of the Philippines, Diliman, Quezon City 1101 Philippines. He is now with the Department of Chemical Engineering, University of Santo Tomas, España Boulevard, Manila 1015 Philippines (e-mail: noel.sabarillo@gmail.com). maximizes their efficiency by reducing their reactivity towards the external environment and controlling volume changes in the storage material during phase change [3].

Various studies have already been established about incorporation of the microencapsulated PCMs (or MEPCMs) in building materials to improve the thermal comfort and incur energy savings [4]; however, only few studies have been done about its application in paints or coatings even though paint coatings are commonly used in buildings and houses. In this study, it is desired to determine the viability of using paint containing $n$-octadecane microencapsulated in resorcinol-modified urea-melamine-formaldehyde (UMF) shells as an LHS (cooling) system for buildings. The paraffin $n$-octadecane $\left(\mathrm{C}_{18} \mathrm{H}_{38}\right)$, with a melting point of $27^{\circ} \mathrm{C}$ [5], is used since it is the most suitable organic PCM to be used for indoor room temperature conditions in the Philippine setting (the average year-round temperature in the Philippines is approximately $26.6^{\circ} \mathrm{C}$ [6]), while UMF is used since it has been reported that the polymer can enhance the chemical and physical stability properties of the $\mathrm{C}_{18} \mathrm{H}_{38}$ [7].

The study also aims to determine the effects of homogenization speed and the dropping rate of the prepolymer solution during synthesis of the microcapsules in the resulting properties of the MEPCMs. The results will provide supplementary information to existing optimization studies about the effects of various possible modifications in the microencapsulation procedure.

\section{Methodology}

\section{A. Emulsion Preparation}

$\mathrm{N}$-octadecane (99 wt\%) and sodium dodecyl sulfate (SDS) were procured from Merck, Inc. (Philippines), while resorcinol was purchased from BE Scientific in Quezon City, Philippines. The reagents were added to $250 \mathrm{~mL}$ of distilled water in a beaker constantly being stirred at $4,500 \mathrm{rpm}$ on top of a hot plate set at $75^{\circ} \mathrm{C}$ for 60 minutes.

\section{B. Pre-polymer Solution Preparation}

Urea and formaldehyde (37 wt \%) were purchased from BE Scientific in Quezon City, Philippines, while melamine (99 wt $\%$ ) was procured from Merck, Inc., Philippines. The reagents were mixed in a beaker and stirred manually before being washed with distilled water. Triethanolamine procured from BE Scientific was added to adjust the $\mathrm{pH}$ of the mixture to 8 , before the mixture was stirred at a constant speed of 500 rpm on a hot plate set at $75^{\circ} \mathrm{C}$.

\section{MEPCM Synthesis}

The method used was adopted from the work of Pilario, 
Reamillo, Sabarillo and Escoto in 2012 [8]. Five minutes before the emulsification was completed, the emulsion was slightly acidified using acetic acid, and then it was stirred at two different mixing speeds (1000 rpm, $4500 \mathrm{rpm})$. The pre-polymer was then added at varying dropping rates $(0.6$ $\mathrm{mL} / \mathrm{min}, 1 \mathrm{~mL} / \mathrm{min}, 3 \mathrm{~mL} / \mathrm{min}$, all at once) to the emulsion, and the resulting mixture was homogenized for 90 minutes. During the synthesis of MEPCMs, the $\mathrm{pH}$ of the mixture was maintained slightly acidic using acetic acid and the temperature was kept constant at $75^{\circ} \mathrm{C}$; afterwards, triethanolamine was added to stop the polymerization.

The microcapsules in the solution were then filtered using a regular filter paper and then washed with $30 \%$ ethanol pre-heated to $50^{\circ} \mathrm{C}$, then further washed with distilled water pre-heated to $90^{\circ} \mathrm{C}$. The filtered microcapsules were put on crucibles and were then dried in a gravity convection oven for about 8 hours.

Table I below summarizes all the samples produced from the synthesis of microcapsules.

TABLE I: SUMMARY OF MICROCAPSULE SAMPLES

\begin{tabular}{lcc}
\hline \multirow{2}{*}{ Dropping rate } & \multicolumn{2}{c}{ Mixing speed } \\
\cline { 2 - 3 } & $4500 \mathrm{rpm}$ & $1000 \mathrm{rpm}$ \\
\hline \hline all at once & $\mathrm{Hmax}$ & $\mathrm{Lmax}$ \\
$3 \mathrm{~mL} / \mathrm{min}$ & $\mathrm{H} 3$ & $\mathrm{~L} 3$ \\
$1 \mathrm{~mL} / \mathrm{min}$ & $\mathrm{H} 1$ & $\mathrm{~L} 1$ \\
$0.6 \mathrm{~mL} / \mathrm{min}$ & $\mathrm{H} .6$ & L.6 \\
\hline \hline
\end{tabular}

\section{MEPCM Characterization}

Chemical characterization was done using Nicolet 6700 Fourier transform infrared (FTIR) spectroscopy, and morphological characterization was done using Hitachi S-3400N Type II scanning electron microscope (SEM).

Thermal characterization was done using TA Instruments Q10 Differential Scanning Calorimeter (DSC). Heating and cooling of the samples were done at a rate of $10^{\circ} \mathrm{C} / \mathrm{min}$ under nitrogen atmosphere. Two heating and cooling cycles were accomplished for each sample. The temperature range for the heating part of the initial cycle of each sample was $10^{\circ} \mathrm{C}$ to $50^{\circ} \mathrm{C}$ while for the rest of the remaining cycles the temperature range was set between $20^{\circ} \mathrm{C}$ and $50^{\circ} \mathrm{C}$.

\section{E. Paint-MEPCM LHS System Synthesis}

In pre-weighed clear vials, white primer enamel paint up were added with the prepared microcapsules at four different concentrations (blank, 1\%, 5\%, 10\%). The paint-MEPCM mixtures were manually homogenized and then immediately applied on the surfaces of $5.1 \mathrm{~cm} \times 5.1 \mathrm{~cm}$ galvanized iron (GI) sheets and were then allowed to dry at ambient temperature overnight. Table II summarizes the samples prepared of the LHS system of paint-MEPCMs.

\section{F. LHS System Thermal Analysis}

The painted GI sheet samples were set up with an improvised temperature data logger system, which was used for temperature profiling. An incandescent light bulb was used to heat the samples in an enclosed, insulated space from $24^{\circ} \mathrm{C}$ to about $42^{\circ} \mathrm{C}$, and then cooled by lifting out the lid with the light bulb and exposing the samples to ambient temperature. Temperature readings were manually recorded every ten seconds.

TABLE II: SUMMARY OF MICROCAPSULE SAMPLES

\begin{tabular}{ccccc}
\hline \hline \multirow{2}{*}{$\begin{array}{c}\text { MEPCM } \\
\text { sample }\end{array}$} & blank & $1 \%$ & $5 \%$ & $10 \%$ \\
\cline { 2 - 5 } Lmax & Lmax-b & Lmax-1 & Lmax-5 & Lmax-10 \\
L3 & L3-b & L3-1 & L3-5 & L3-10 \\
L1 & L1-b & L1-1 & L1-5 & L1-10 \\
L0.6 & L0.6-b & L0.6-1 & L0.6-5 & L0.6-10 \\
Hmax & Hmax-b & Hmax-1 & Hmax-5 & Hmax-10 \\
H3 & H3-b & H3-1 & H3-5 & H3-10 \\
H1 & H1-b & H1-1 & H1-5 & H1-10 \\
H.6 & H.6-b & H.6-1 & H.6-5 & H.6-10 \\
\hline \hline
\end{tabular}

\section{RESULTS AND DISCUSSION}

\section{A. FTIR and SEM Analysis of MEPCMs}

As shown in Fig. 1 and Fig. 2, the FTIR spectra of the eight MEPCMs samples are all similar: the peaks that are present in each spectrum are almost the same and comparable.

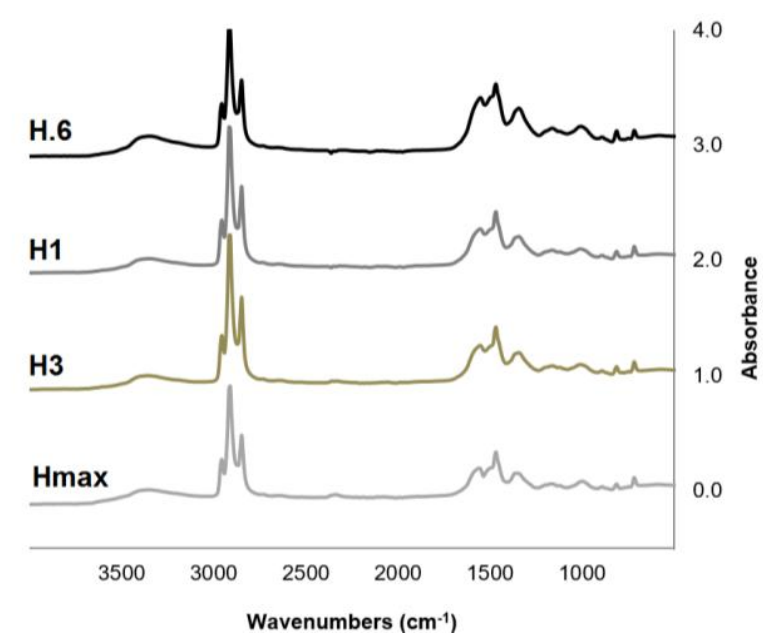

Fig. 1. FTIR spectra for MEPCM samples stirred at $4500 \mathrm{rpm}$.

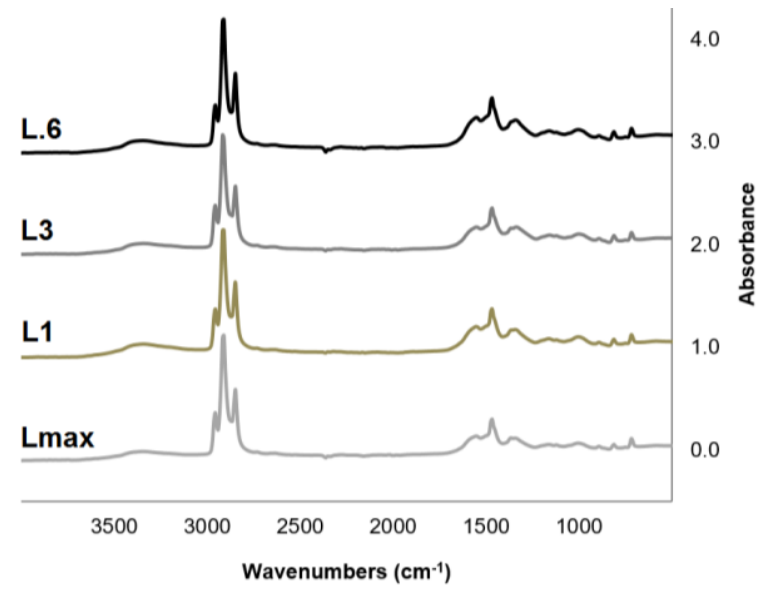

Fig. 2. FTIR spectra for MEPCM samples stirred at $1000 \mathrm{rpm}$.

The peak at around $3300 \mathrm{~cm}^{-1}$ corresponds to the hydroxyl $(\mathrm{O}-\mathrm{H})$, imino $(\mathrm{C}=\mathrm{N})$ and amino $(\mathrm{N}-\mathrm{H})$ stretching [9]. The peaks in the range of $2900 \mathrm{~cm}^{-1}$ relate to $\mathrm{C}-\mathrm{H}$ stretching vibrations [10]. The peaks that are positioned near the 1550 $\mathrm{cm}^{-1}$ wavenumber suggest $\mathrm{C}-\mathrm{N}$ multiple stretching in the triazine ring. The peaks at around $1350 \mathrm{~cm}^{-1}$ and $1460 \mathrm{~cm}^{-1}$ 
can be attributed to the $\mathrm{C}-\mathrm{H}$ bending vibrations in the methylene group. The absorption peaks at the proximity of $1100 \mathrm{~cm}^{-1}$ suggest aliphatic $\mathrm{C}-\mathrm{N}$ vibration. The $810 \mathrm{~cm}^{-1}$ peaks, they pertain to characteristic triazine bending, while the ones at $715 \mathrm{~cm}^{-1}$ range imply in-plane rocking vibration of the methylene group. The presence of these peaks in all FTIR spectra confirms that $n$-octadecane is successfully microencapsulated into the UMF polymer shell [11], [12]. Moreover, there are also broad peaks at around $3390 \mathrm{~cm}^{-1}$ that are overlapped by the imino and amino stretching peaks, and those peaks correspond to phenolic hydroxyl stretching vibrations. The peaks in the $1550 \mathrm{~cm}^{-1}$ and $1100 \mathrm{~cm}^{-1}$ that are mentioned earlier also could be attributed to vibrations of the phenol ring and stretching vibrations of the $\mathrm{C}-\mathrm{O}$ in the phenyl ring- these absorption peaks indicates that resorcinol has cross-linked to the polymer shell [13].

SEM images shown in Fig. 3 reveal the surface morphology and size of the MEPCMs synthesized at different mixing speeds and pre-polymer dropping rates. The average sizes of the MEPCM capsules are listed in Table III. It can be observed that the microcapsules produced at a stirring rate of $4500 \mathrm{rpm}$ are smaller compared to those synthesized at a stirring rate of $1000 \mathrm{rpm}$. This is because increasing the stirring rate results to an increase in shear forces acting on the emulsion, resulting to smaller particles that have higher surface energy, making them more thermodynamically unstable and with a stronger tendency to agglomerate [14], [15].

TABLE III: AVERAGE DIAMETERS OF MEPCMS (IN MICROMETERS)

\begin{tabular}{ccc}
\hline \hline & \multicolumn{2}{c}{ Mixing speed } \\
\cline { 2 - 3 } Dropping rate & $4500 \mathrm{rpm}$ & $1000 \mathrm{rpm}$ \\
\hline all at once & 45.7 & 336 \\
$3 \mathrm{~mL} / \mathrm{min}$ & 33.4 & 307 \\
$1 \mathrm{~mL} / \mathrm{min}$ & 23.7 & 235 \\
$0.6 \mathrm{~mL} / \mathrm{min}$ & 21.1 & 99.9 \\
\hline \hline
\end{tabular}

It could also be observed that the size of the MEPCMs decreases as the dropping rate decreases. Moreover, it has been observed that the distribution of particles becomes narrower as the stirring rate and dropping rate decreases. A narrow size distribution is desired because it is one of the factors that indicate the applicability of MEPCMs since some applications have certain size requirements [16], [17].

The observed effect of the dropping rate to the size of the particles can be explained by the microencapsulation mechanism of the $n$-octadecane, which involves formation of micelles through dispersion of $n$-octadecane particles in water with the aid of anionic SDS [18]. From this emulsification, the $n$-octadecane micelles will gain negative charges due to the hydrophilic sulfate ion, while the methylolation products of melamine or urea with the formaldehyde will gain positive charges due to the acidified environment [19]. The difference in charges between the molecules causes the pre-polymer molecules to deposit onto the surface of the micelles. Therefore, if the dropping of the pre-polymer is slow enough, there will be sufficient time for the pre-polymer molecules to be sheared into small discrete amounts and be evenly dispersed in the solution with the agitated $n$-octadecane, resulting to finer particles. On the other hand, due to the high charge density delivered by the SDS, if the dropping rate is high or the stirring rate is low, the pre-polymer molecules may agglomerate and polymerize too soon before they can reach and be dispersed along the surfaces of the micelles. Due to the highly negative charge of the sulfate ion of the micelles, the ionic interaction with the positively charged UMF may overcome the shear forces applied by the homogenizer, leading to non-uniform distribution of prepolymer molecules on the surface of the n-octadecane and larger particles. This is consistent and analogous with the results of Lone, et al. in 2013 about the effect of flowrate on the microcapsule particle size [19].
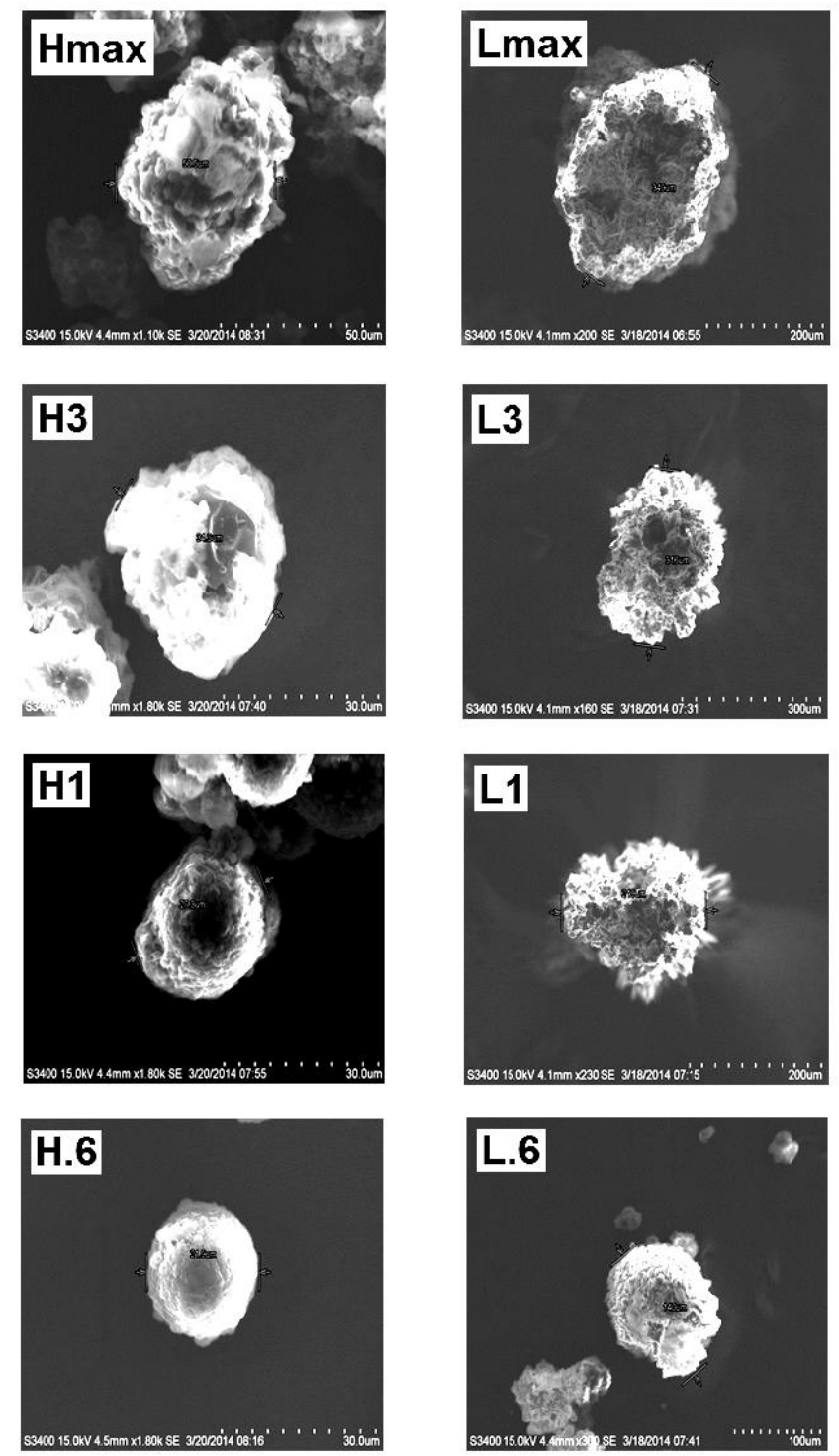

Fig. 3. SEM images of the MEPCM samples stirred at different mixing speeds and at pre-polymer dropping rates.

\section{B. DSC Analysis of MEPCMs}

The MEPCMs are characterized using DSC to determine the effect of varying mixing speed and dropping rates on the thermal properties of the samples. The resulting thermograms for the microcapsules are also compared to that of pure (99\%) $n$-octadecane. Fig. 4 shows representative thermograms of MEPCM samples prepared at different mixing speeds in 
comparison to that of pure $n$-octadecane, and Tables IV and V summarizes the thermal data and latent heat values derived from the DSC characterization.
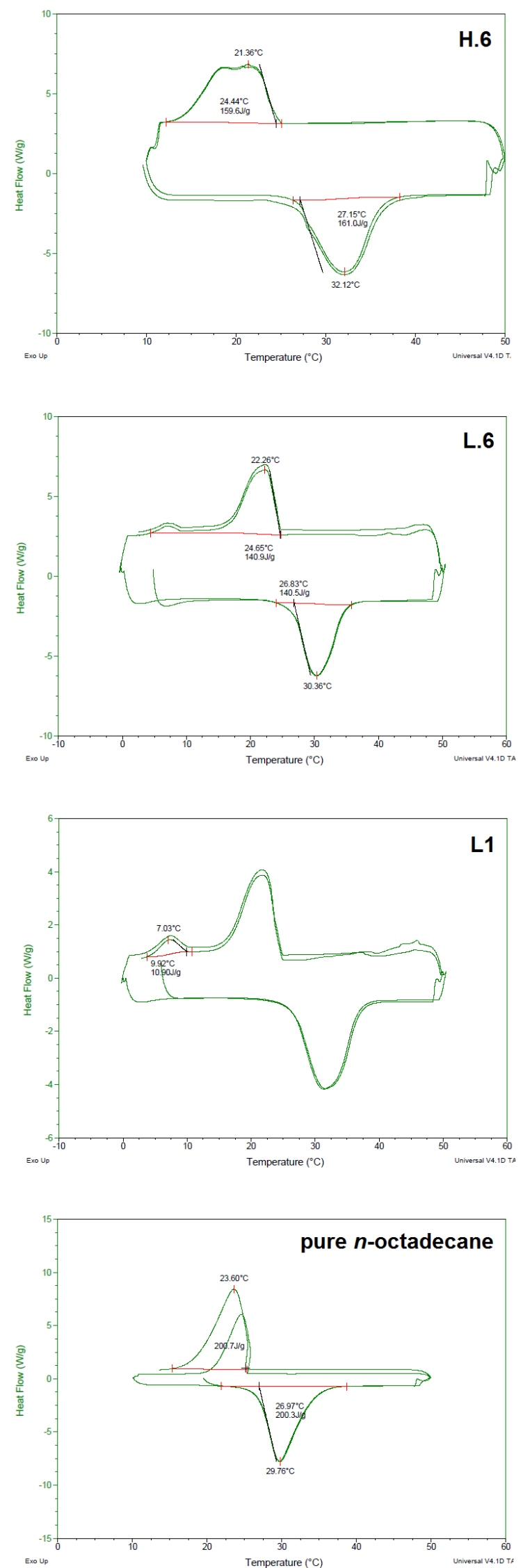

Fig. 4. DSC thermograms for MEPCM samples and for pure $n$-octadecane.

From the thermogram results, it can be noticed that the measured properties for sample H1 (onset melting temperature, peak melting temperature, onset crystallization temperature, peak crystallization temperature) are slightly off compared to the those measured from the other samples, which may possibly be due to the presence of residual un-encapsulated $n$-octadecane in the microcapsules.

It can also be observed from Fig. 4 that for sample L1, two peaks can be observed from the cooling region of the thermogram cycles. These two peaks correspond to peaks $\alpha$ and $\beta$ which correspond to heterogeneous liquid-rotator transition and homogeneous liquid-crystal transition, respectively [13]. The $\alpha$ peak corresponds to a higher temperature because less energy is needed to achieve the heterogeneous liquid-rotator transition compared to the energy required for homogeneous liquid-crystal transition.

TABLE IV: THERMAL DATA OBTAINED FROM THE DSC ANALYSIS

\begin{tabular}{|c|c|c|c|c|}
\hline \multirow[b]{2}{*}{$\begin{array}{l}\text { MEPCM } \\
\text { sample }\end{array}$} & \multicolumn{4}{|c|}{ Thermal Properties } \\
\hline & $\begin{array}{l}\text { Onset } \\
\text { melting } \\
\text { temperature } \\
\left({ }^{\circ} \mathrm{C}\right)\end{array}$ & $\begin{array}{l}\text { Peak } \\
\text { melting } \\
\text { temperature } \\
\left({ }^{\circ} \mathrm{C}\right)\end{array}$ & $\begin{array}{l}\text { Onset } \\
\text { crystallization } \\
\text { temperature } \\
\left({ }^{\circ} \mathrm{C}\right)\end{array}$ & $\begin{array}{l}\text { Peak } \\
\text { crystallization } \\
\text { temperature } \\
\left({ }^{\circ} \mathrm{C}\right)\end{array}$ \\
\hline Lmax & - & - & - & - \\
\hline L3 & 27.23 & 31.62 & 24.05 & $20.97,6.22$ \\
\hline L1 & 26.68 & 31.66 & 24.24 & $21.75,7.03$ \\
\hline L0.6 & 26.87 & 30.43 & 24.65 & $22.26,6.67$ \\
\hline Hmax & 27.03 & 33.64 & 24.62 & $19.96,5.53$ \\
\hline H3 & 26.91 & 31.80 & 24.73 & 20.21 \\
\hline $\mathrm{H} 1$ & 27.36 & 37 & 25.04 & 15.31 \\
\hline H.6 & 27.15 & 32.12 & 24.44 & 21.36 \\
\hline $\begin{array}{c}\text { pure } \\
n-\mathrm{C}_{18} \mathrm{H}_{38}\end{array}$ & 26.97 & 29.76 & 25 & 23.6 \\
\hline
\end{tabular}

TABLE V: LATENT HEAT VALUES FROM DSC ANALYSIS

\begin{tabular}{ccc}
\hline \hline \multirow{2}{*}{$\begin{array}{c}\text { MEPCM } \\
\text { sample }\end{array}$} & \multicolumn{2}{c}{ Thermal Properties } \\
\cline { 2 - 3 } Lmax & $\begin{array}{c}\text { Latent heat of fusion } \\
\text { melting }(\mathrm{J} / \mathrm{g})\end{array}$ & $\begin{array}{c}\text { Latent heat of fusion } \\
\text { crystallization }(\mathrm{J} / \mathrm{g})\end{array}$ \\
\hline L3 & 151.7 & - \\
L1 & 152.6 & 95.98 \\
L0.6 & 155.6 & 112.6 \\
$\mathrm{Hmax}$ & 141.2 & 121.5 \\
$\mathrm{H} 3$ & 164.8 & 118.2 \\
$\mathrm{H} 1$ & 171.8 & 145.2 \\
$\mathrm{H} .6$ & 175.8 & 153.5 \\
pure $n-\mathrm{C}_{18} \mathrm{H}_{38}$ & 200.3 & 200.7 \\
\hline \hline
\end{tabular}

Aside from the thermal properties, the core-to-shell ratio of the microcapsules can also be determined based from the latent heat of fusion $\left(\Delta H_{m}\right)$ values of the samples by calculating the encapsulation ratio, $R$, using the formula:

$$
R=\frac{\Delta H_{m, \mathrm{MEPCM}}}{\Delta H_{m, n-\mathrm{C}_{18} \mathrm{H}_{38}}}
$$


The value of $R$ indicates the heat of fusion of the encapsulated $n-\mathrm{C}_{18} \mathrm{H}_{38}$ relative to that of pure $n-\mathrm{C}_{18} \mathrm{H}_{38}$, and is equivalent to the weight fraction of $n-\mathrm{C}_{18} \mathrm{H}_{38}$ encapsulated in the MEPCM.

For the samples synthesized at a mixing speed of $4500 \mathrm{rpm}$, the values of latent heat of fusion for both melting and crystallization generally decreases as the dropping rate increases, which is due to the poor dispersion of pre-polymer molecules on the surface of the $n$-octadecane micelles with increasing dropping rate, resulting to thicker shell structure. It can also be observed that these samples have higher peak melting temperatures and lower peak crystallization temperatures compared to the values measured for the pure $n$-octadecane, which could be attributed to the low thermal conductivity of $n$-octadecane and the insulating effect of the thin UMF shell.

The feed core-to-shell ratio (wt/wt) for this study is set at $70 / 30$ but, as shown in Table VI, this value will not necessarily be the attained on the actual samples. The deviation in the core-to-shell ratios is probably because of the presence of un-encapsulated $n$-octadecane, and because not all pre-polymer molecules has adhered to the surface of the $n$-octadecane particles. Also, from the computed values, it can be observed that $R$ decreases with increasing dropping rate, which indicates an overall increase in the amount of microencapsulated PCM. This may be expected since an increase in dropping rate means an increase in the number of pre-polymer molecules in the emulsion that will consequently form the microcapsules.

\begin{tabular}{ccc}
\multicolumn{2}{c}{ TABLE VI: SUMMARY OF CALCULATED ENCAPSULATION RATIOS } \\
\hline \hline MEPCM & \multicolumn{2}{c}{ Encapsulation ratio, $R$} \\
\cline { 2 - 3 } sample & Feed & Actual \\
\hline \hline Lmax & $70 / 30$ & - \\
L3 & $70 / 30$ & $76 / 24$ \\
L1 & $70 / 30$ & $76 / 24$ \\
L0.6 & $70 / 30$ & $78 / 22$ \\
Hmax & $70 / 30$ & $70 / 30$ \\
H3 & $70 / 30$ & $82 / 18$ \\
H1 & $70 / 30$ & $85 / 14$ \\
H.6 & $70 / 30$ & $88 / 12$ \\
\hline \hline
\end{tabular}

\section{Paint-MEPCM LHS System Thermal Analysis}

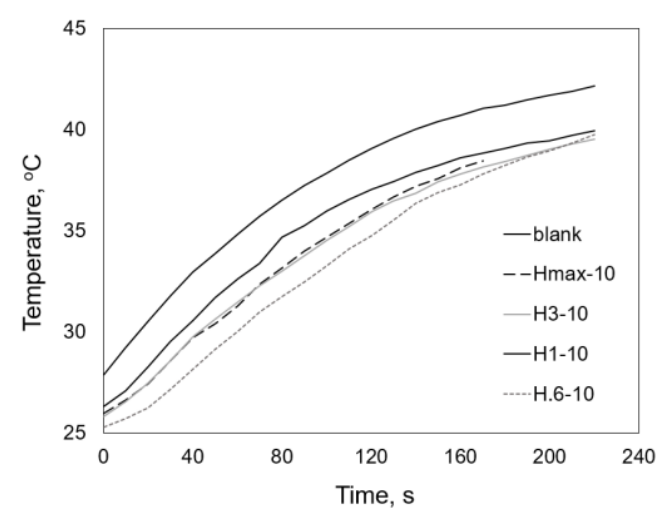

Fig. 5. Temperature profiles for paint-MEPCM with 10\% MEPCM synthesized at a mixing speed of $4500 \mathrm{rpm}$.

The representative temperature profiles of the LHS system samples heated using incandescent light bulbs are shown below in Fig. 5, Fig. 6, Fig. 7 and Fig. 8, and the representative values are enumerated in Table VII, Table VIII and Table IX. The slope difference between the blank and the paint-MEPCM samples is set as the basis of analysis as this is an indication of the thermal buffering capacity of the paint-MEPCM systems.

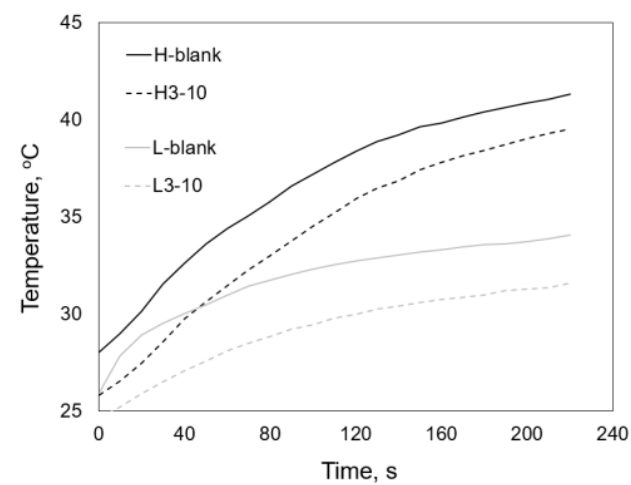

Fig. 6. Temperature profiles for paint-MEPCM with 10\% MEPCM synthesized at mixing speeds of $1000 \mathrm{rpm}$ and $4500 \mathrm{rpm}$.

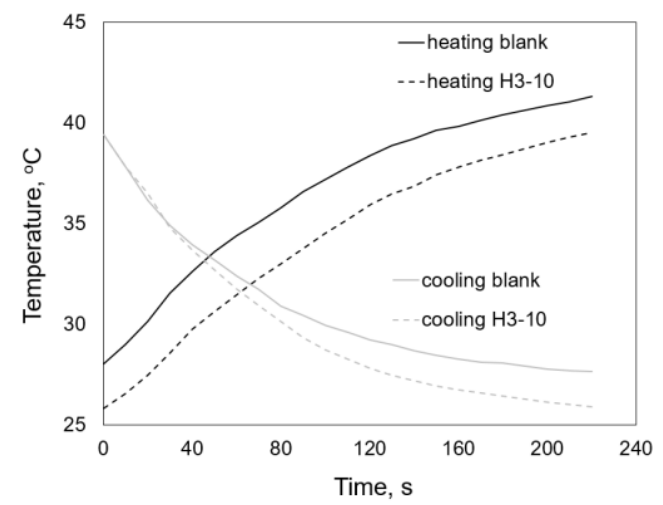

Fig. 7. Heating and cooling temperature profiles for paint-MEPCM with $10 \%$ MEPCM synthesized at a mixing speed of $4500 \mathrm{rpm}$.

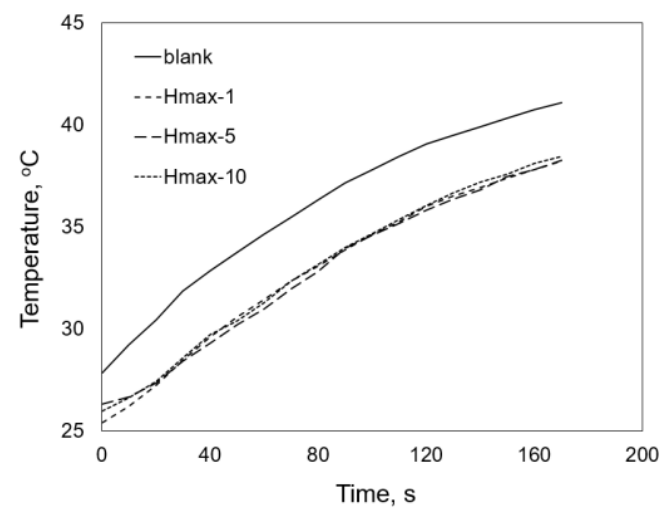

Fig. 8. Temperature profiles for paint-MEPCM with $1 \%, 5 \%$, and $10 \%$ MEPCM synthesized at mixing speeds of $4500 \mathrm{rpm}$.

TABLE VII: COMPARISON OF HEATED SAMPLE WITH 10\% MEPCMS SYNTHESIZED AT A MIXING SPEED OF 4500 RPM

\begin{tabular}{cc}
\hline $\begin{array}{c}\text { Paint-MEPCM } \\
\text { sample }\end{array}$ & $\begin{array}{c}\text { Slope difference between the blank and } \\
\text { the sample from } 28-35^{\circ} \mathrm{C}\left(\text { in }{ }^{\circ} \mathrm{C} / \mathrm{s}\right)\end{array}$ \\
\hline \hline Hmax-10 & 0.039 \\
H3-10 & 0.013 \\
H1-10 & 0.028 \\
H.6-10 & 0.024 \\
\hline \hline
\end{tabular}


The temperature profiles illustrate the differences in the rates of temperature increase between the blank sample and sample with different MEPCMs concentration in the aforementioned temperature range. The temperature range of $28^{\circ} \mathrm{C}$ to $35^{\circ} \mathrm{C}$ is chosen because it best represents the indoor temperature range during a typical daytime in the Philippines.

TABLE VIII: COMPARISON OF HEATED SAMPLE WITH 10\% MEPCMS SYNTHESIZED AT A MIXING SPEED OF 1000 RPM AND 4500 RPM

\begin{tabular}{cc}
\hline $\begin{array}{c}\text { Paint-MEPCM } \\
\text { sample }\end{array}$ & $\begin{array}{c}\text { Slope difference between the blank and } \\
\text { the sample from } 28-35^{\circ} \mathrm{C}\left(\text { in }{ }^{\circ} \mathrm{C} / \mathrm{s}\right)\end{array}$ \\
\hline \hline L1-10 & 0.019 \\
L0.6-10 & 0.005 \\
H1-10 & 0.028 \\
H.6-10 & 0.024 \\
\hline \hline
\end{tabular}

TABLE IX: COMPARISON OF HEATED SAMPLE WITH 1\%, 5\% AND 10\% MEPCMS SYNTHESIZED AT A MIXING SPEED OF 4500 RPM

\begin{tabular}{cc}
\hline $\begin{array}{c}\text { Paint-MEPCM } \\
\text { sample }\end{array}$ & $\begin{array}{c}\text { Slope difference between the blank and } \\
\text { the sample from } 28-35^{\circ} \mathrm{C}\left(\text { in }{ }^{\circ} \mathrm{C} / \mathrm{s}\right)\end{array}$ \\
\hline \hline Hmax-1 & 0.039 \\
Hmax-5 & 0.032 \\
Hmax-10 & 0.003 \\
\hline \hline
\end{tabular}

For the comparison of samples containing 10\% MEPCMs synthesized at $4500 \mathrm{rpm}$ at different pre-polymer solution dropping rates, as pre-polymer dropping rate decreases, the temperature buffering capacity unexpectedly decreases. This is because at any given concentration, more heat can be absorbed by the system for the same concentration of MEPCMs as the latent heat $(\mathrm{J} / \mathrm{g})$ of the microcapsules increases- thus, resulting to better temperature buffering capacity. One possible explanation for this is that either more microcapsules (concentration $>10 \%$ ) have been painted on the surface of sample Hmax-10 or less microcapsules (concentration $<10 \%$ ) have been deposited on the surfaces of samples H3-10, H1-10 and H.6-10 during the manual incorporation and mixing of the paint-MEPCM systems. An increase in the number of microcapsules with respect to the desired $10 \%$ concentration will cause an increase in the total latent heat that can be absorbed by the system, thereby increasing the temperature buffering capacity of the system. On the other hand, having deposited less number of microcapsules on the surface of the samples will lead to a decrease in the total latent heat that can be absorbed by the system, effectively decreasing its buffering capacity [20].

For the comparison of samples at varying homogenization speeds, it can be deduced from Table VIII that mixing done at a higher speed results to better temperature buffering capacity to the painted samples compared to the samples synthesized at $1000 \mathrm{rpm}$, which agrees well with the observed values in the latent heat and encapsulation ratio of the samples. Samples H1 and H.6 have higher latent heats and encapsulation ratios compared to samples L1 and L.6, respectively, and this would consequently result to higher temperature buffering capacity for samples $\mathrm{H} 1$ and H.6, as explained previously.

For the comparison of samples with various MEPCM concentrations, it is observed that with increasing MEPCM concentration ( $1 \%$ to $10 \%$ ) the buffering capacity of the system also increases. This can be attributed to the total latent heat that can be absorbed by the system. As the concentration of microcapsules increases, the total latent heat that can be absorbed also increases, thus resulting to better temperature buffering of the system. Lastly, the temperature differences between the blank samples and the paint with MEPCM samples for all the samples characterized fall approximately in the range of $1{ }^{\circ} \mathrm{C}$ to $4^{\circ} \mathrm{C}$. These results imply that the paint-MEPCMs have successfully buffered or delayed the increase in temperature of the samples.

\section{CONCLUSIONS AND RECOMMENDATIONS}

Increasing the homogenization speed at constant pre-polymer dropping rate decreases the size of the microcapsules. Also, decreasing the dropping rate of pre-polymer solution at constant homogenization speed would result to smaller and more spherical microcapsules. DSC results determined the thermal properties and the actual core-to-shell or encapsulation ratio of the MEPCMs. The onset melting temperatures of the sample fall in the range of $26.68^{\circ} \mathrm{C}$ to $27.36^{\circ} \mathrm{C}$ while the peak melting temperature lie between $30.43^{\circ} \mathrm{C}$ to $33.64^{\circ} \mathrm{C}$. The latent heats and encapsulation ratio of the MEPCM were found to increase with increasing mixing speed at constant pre-polymer dropping rate. On the other hand, decreasing the pre-polymer solution dropping rate at constant homogenization speeds increases the latent heat and encapsulation ratio of the samples. The values of latent heat of the samples lie in the range $118.2 \mathrm{~J} / \mathrm{g}$ to $159.6 \mathrm{~J} / \mathrm{g}$ while the encapsulation ratios of the samples range from $60 \%$ to $80 \%$.

Temperature profiling of the paint-MEPCM mixture on GI sheets shows that decreasing the pre-polymer solution dropping rate at constant homogenization speed and MEPCM concentration decreases the temperature buffering capacity of the system. On the other hand, increasing the mixing speed in the synthesis of MEPCMs at any given pre-polymer dropping rate and MEPCM concentration increases the temperature buffering capacity of the LHS system. Lastly, increasing the concentration of MEPCMs at the same homogenization speed and pre-polymer dropping rate also increases the system temperature buffering capacity.

For future studies, it is recommended that a PCM with a slightly higher phase change temperature be used in the synthesis of LHS systems. It is also recommended that focus be given on the effect of other modifications in the microencapsulation procedure on the resulting properties of the microcapsules. Determination of the thermal stability of the microcapsules through several heating and cooling cycles, and optimization studies are also recommended to be done for future researches.

\section{REFERENCES}

[1] D. Zhou, C.Y. Zhao, and Y. Tian, "Review on thermal energy storage with phase change materials (PCMs) in building applications," Applied Energy, vol. 92, pp. 593-605, 2012.

[2] M. Farid, A. Khudhair, S. Razack, and S. Al-Hallaj, "A review on phase change energy storage: materials and applications," Energy Conversion and Management, vol. 45, no. 9-10, pp. 1597-1615, 2004. 
[3] C. Zhao and G. Zhang, "Review on microencapsulated phase change materials (MEPCMs): fabrication, characterization and applications,' Renewable and Sustainable Energy Reviews, vol. 15, no. 8, pp. 3813-3832, 2011.

[4] F. Kuznika, D. Davida, K. Johannes, and J.-J. Roux, "A review on phase change materials integrated in building walls," Renewable and Sustainable Energy Reviews, vol. 15, pp. 379-391, 2011.

[5] C. Velez, M. Khayet, and J. M. Ortiz de Zárate, "Temperaturedependent thermal properties of solid/liquid phase change evennumbered n-alkanes: n-hexadecane, n-octadecane and n-eicosane," Applied Energy, vol. 143, pp. 383-394, 2015.

[6] PAGASA. (June 25, 2013). Climate of the Philippines. [Online]. Available: http://kidlat.pag-asa.dost.gov.ph/cab/statfram.htm

[7] L. Sanchez-Silva, J. F. Rodriguez, M. Carmona, A. Romero, and P. Sanchez, "Thermal and morphological stability of polystyrene microcapsules containing phase-change materials," Journal of Applied Polymer Science, vol. 120, pp. 291-297, 2011.

[8] K. E. S. Pilario, J. G. S. Reamillo, N. S. Sabarillo, and A. D. Escoto, "Synthesis and characterization of microencapsulated n-octadecane in urea- melamine-formaldehyde shell for thermal regulation," Philippine Engineering Journal, vol. 33, no. 1, pp. 69-75, 2012.

[9] C. Gong, H. Zhang, and X. Wang, "Effect of shell materials on microstructure and properties of microencapsulated n-octadecane," Iranian Polymer Journal, vol. 18, no. 6, pp. 501-512, 2009.

[10] J. F. Su, "Synthesis of polyurethane microPCMs containing noctadecane by interfacial polycondensation: influence of styrenemaleic anhydride as a surfactant," Colloids and Surfaces A: Physicochem. Eng. Aspects, vol. 229, pp. 268-275, 2007.

[11] W. Li, J. Wang, X. Wang, S. Wu, and X. Zhang, "Effects of ammonium chloride and heat treatment on residual formaldehyde contents of melamine-formaldehyde microcapsules," Colloid Polym. Sci., vol. 285, no. 15, pp. 1691-1697, 2007.

[12] X. Zhang, Y. Fan, X. Tao, and K. Yick, "Crystallization and prevention of supercooling of microencapsulated n-alkanes," Journal of Colloid and Interface Science, vol. 281, pp. 299-306, 2005.

[13] H. Zhang and X. Wang, "Fabrication and performances of microencapsulated phase change materials based on n-octadecane core and resorcinol-modified melamine-formaldehyde shell," Colloids and Surfaces A: Physicochemical and Engineering Aspects, vol. 332, pp. 129-138, 2009

[14] B. Duan, "Microencapsulation via In Situ Polymerization," in Handbook of Encapsulation and Controlled Release, M. Mishra, Ed. FL: CRC Press, 2016, pp. 307-317.

[15] W. Li, X. Zhu, N. Zhao, and Z. Jiang, "Preparation and properties of melamine urea-formaldehyde microcapsules for self-healing of cementitious materials," Materials, vol. 9, pp. 152-168, 2016.

[16] V. Tyagi, S. Kaushik, S. Tyagi, and T. Akiyama, "Development of phase change materials based microencapsulated technology for buildings: a review," Renewable and Sustainable Energy Reviews, vol. 15, pp. 1373-1391, 2011.

[17] N. Sarier and E. Onder, "Organic phase change materials and their textile applications: an overview," Thermochimica Acta, vol. 540, pp. 7-60, 2012.

[18] M. Nomura, H. Tobita, and K. Suzuki, "Emulsion Polymerization: Kinetic and Mechanistic Aspects," Advances in Polymer Science, vol. 175, pp. 1-128, 2005.
[19] S. Lone, H. M. Lee, G. M. Kim, W.-G. Koh, and I. W. Cheong, "Facile and highly efficient microencapsulation of a phase change material using tubular microfluidics," Colloids and Surfaces A: Physicochem. Eng. Aspects, vol. 422, pp. 61-67, 2013.

[20] N. S. Sabarillo, "Characterization of latent heat storage system of MEPCM-incorporated paint,” M.S. thesis, Dept. Chem. Eng., Univ. of the Philippines Diliman, Quezon City, Philippines, 2013.

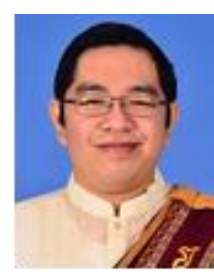

Terence P. Tumolva was born in February 1982 in Quezon City. Dr. Tumolva studied at the University of the Philippines Diliman in Quezon City, Philippines from 1998 to 2003, where he earned his bachelor of science degree in chemical engineering, and then pursued and earned his master of science degree in chemical engineering at University of the Philippines Diliman in 2006. He then went on to study at Tokyo Institute of Technology under the Monbukagakusho (Japanese Government) Scholarship program in 2007, where he earned his doctor of engineering degree in chemical engineering in 2011.

Dr. Tumolva started working as an instructor at the Department of Chemical Engineering in the University of the Philippines Diliman in 2003 where he continued to teach and conduct research as an assistant professor since July 2006, and then as an associate professor from January 2014 up to the present. He also served as the college secretary of the College of Engineering at the University of the Philippines Diliman from June 2011 to August 2016, and is currently serving as Chair of the Department of Chemical Engineering at the University of the Philippines Diliman since September 2016. He is a licensed chemical engineer (registered by the Professional Regulations Commission) in the Philippines and an active member of the Philippine Institute of Chemical Engineers Metro Manila Academe Chapter (PIChE-MMAC) since 2011. He is also a senior member of the Asia-Pacific Chemical, Biological and Environmental Engineering Society (APCBEES) since March 2016. He has published several journal papers and presented many international conference papers in green composites and green materials technology.

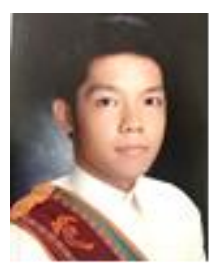

Noel S. Sabarillo was born in December 1991 in Parañaque City, Philippines. Engr. Sabarillo studied at the University of the Philippines Diliman in Quezon City, Philippines from June 2007 to April 2012, where earning his bachelor of science degree in chemical engineering. He then pursued his master of science degree in chemical engineering from the University of the Philippines Diliman under the Engineering Research and Development for Technology (ERDT) Scholarship program of the Department of Science and Technology (DOST) in the Philippines from June 2012 to April 2014.

Engr. Sabarillo worked as an associate professor at Far Eastern University Institute of Technology in Manila, Philippines from September 14 to November 2016 and is currently an instructor at the Department of Chemical Engineering in the University of Santo Tomas since November 2014. He is a licensed chemical engineer (registered by the Professional Regulations Commission) in the Philippines and a member of the Philippine Institute of Chemical Engineers Metro Manila Academe Chapter (PIChE-MMAC). 